\title{
A Role for the Epithelial Microenvironment at Tumor Boundaries
}

\section{Evidence from Drosophila and Human Squamous Cell Carcinomas}

\author{
Marcos Vidal, ${ }^{*}$ Lorena Salavaggione, ${ }^{\dagger}$ \\ Lourdes Ylagan, ${ }^{\ddagger}$ Mark Wilkins, ${ }^{\S}$ Mark Watson, ${ }^{\dagger}$ \\ Katherine Weilbaecher, ${ }^{\S}$ and Ross Cagan ${ }^{\text {T }}$ \\ From the Beatson Institute for Cancer Research, ${ }^{*}$ Cancer \\ Research United Kingdom, Glasgow, United Kingdom; the \\ Division of Laboratory Medicine, ${ }^{\dagger}$ the Departments of \\ Pathology, ${ }^{\ddagger}$ and Medicine, Division of Oncology, ${ }^{\circledR}$ Washington \\ University School of Medicine, St. Louis, Missouri; and the \\ Department of Developmental and Regenerative Biology, " \\ Mount Sinai School of Medicine, New York, New York
}

Recent work has shown an increasing appreciation for the importance of the tumor environment, most commonly the overlying stroma. Less emphasis has been placed on the importance of local communication between transformed cells and their neighbors within the epithelium at tumor boundaries. We previously reported a Drosophila model that highlighted the importance of local interactions within the epithelial microenvironment: Src-transformed cells (Csk-deficient) were influenced by their immediate normal neighbors. The result was a consistent change in 'border cells' at the edge of transformed patches including delocalized p120-catenin and E-cadherin as well as invasive migration through the basal lamina. Here we show that the invasive properties of the boundary cells depend on up-regulation of Drosophila matrix metalloproteinase-1 as assessed by promoter activity, protein levels, in situ enzymatic activity, and tests of genetic modifier activity. Further, we provide evidence that these events at tumor borders may be evolutionarily conserved. We detected changes in 'boundary cells' within histological sections of human squamous cell carcinomas that were similar to those observed in Drosophila: both E-cadherin and p120-catenin exhibited normal junctional localization at the centers of the tumors but were reduced or delocalized at the boundary. Further, matrix metalloproteinase- 2 was up regulated within these same boundary cells. These results support the view that local cell-cell interactions within the epithelial microenvironment impact tumor invasion and progression. (Am J Patbol 2010, 176:3007-3014; DOI: 10.2353/ajpath.2010.090253)

Recent studies have highlighted the importance of interactions between tumors and their environment. Factors from the local microenvironment including extracellular matrix components, fibroblasts, and macrophages and other immune cells can influence the behavior of cancer cells both in terms of growth and metastasis. ${ }^{1}$ Less understood is the influence of local interactions between transformed cells and their epithelial neighbors. These interactions are difficult to study because they occur early in tumor progression, and most human tumors are identified after tumors have significantly advanced. Recently, we presented data from a Drosophila tumor model that suggested the importance of early, local cell-cell interactions within the epithelium. ${ }^{2}$ Here, we further explore this model and in addition offer evidence for this view from human oral squamous cell carcinomas (SCCs).

The effects of the microenvironment are best studied in vivo. One system that offers the opportunity to study the effects of the microenvironment in situ is the fruit fly Drosophila. Fly models offer powerful genetic tools and a genome that contains orthologs of a majority of known oncogenes and tumor suppressors. As such, Drosophila is becoming increasingly popular as a cancer model (reviewed in 3,4). Relevant to this article,

Supported by NIH grants R01-CA109730 and R01-CA084309 from the National Cancer Institute, Department of Defense DOD-W81XWH-07-10360, and Cancer Research UK.

Accepted for publication February 16, 2010

Address reprint requests to Marcos Vidal, Ph.D., Beatson Institute for Cancer Research, Garscube Estate, Switchback Road B, Cancer Research UK, Glasgow, UK. E-mail: m.vidal@beatson.gla.ac.uk.. 

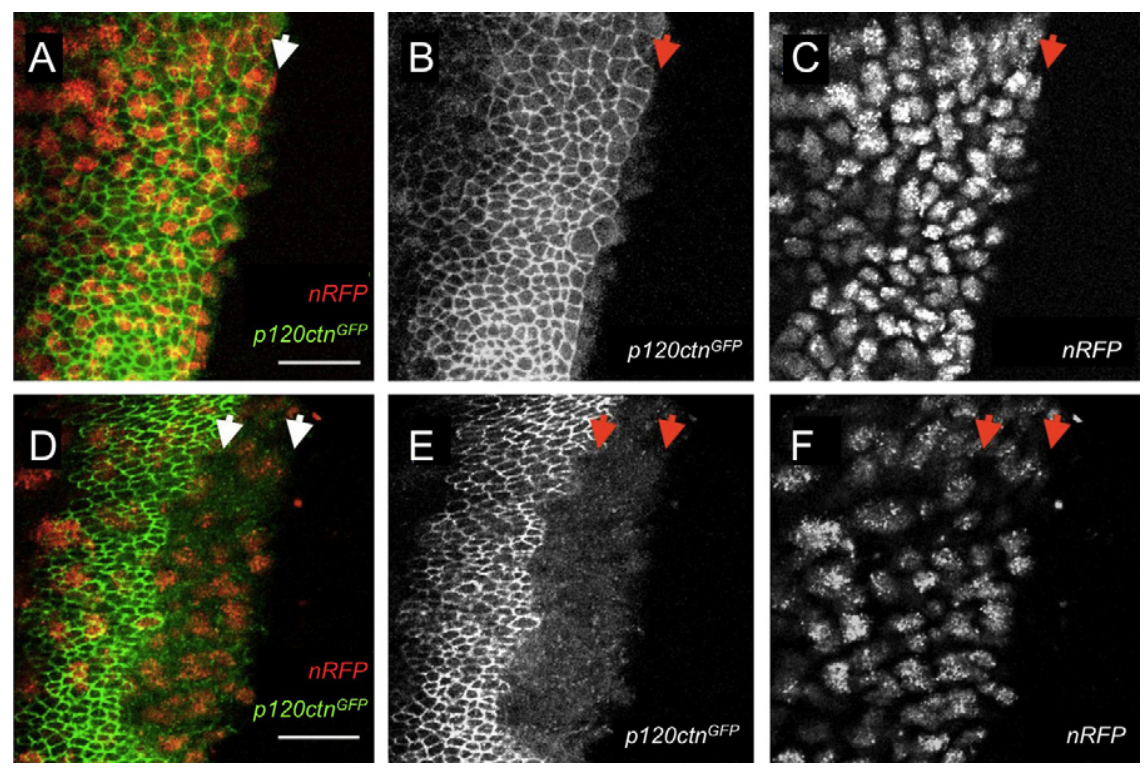

Figure 1. Junctions were disrupted at the border of $d C s k-I R$ patches. The $p t c$ promoter was used to express transgenes in a discrete stripe across the long-axis of the developing wing disk, a monolayer epithelium. Specifically, junctions were labeled by dP120-catenin-GFP (green in $\mathbf{A}$ and $\mathbf{D}$ gray in $\mathbf{B}$ and $\mathbf{E}$ ); ptc-positive cells were independently labeled with the nuclear marker RFP $[n l s]$ (red in $\mathbf{A}$ and $\mathbf{D}$; gray in $\mathbf{C}$ and $\mathbf{F}$ ). Neighboring wild-type cells were unlabeled (right-most region of each panel). A-C: In control wing disks

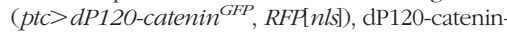
GFP-labeled adherens junctions were observed in all cells within the patched region. Arrows indicate the anteroposterior boundary. D-F: As shown previously, ${ }^{2} d C s k$-deficient boundary cells ( $p t c>$

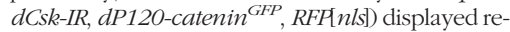
duced or delocalized dP120-catenin (area between the two arrows). The confocal projections ranged from the apical adherens junctions to the nuclei of wing disks from Drosophila larvae. Scale bars $=20 \mu \mathrm{m}$

the Drosophila tumor suppressor ortholog C-terminus Src Kinase (dCsk) was identified both as a factor mediating activity of the Lats/Warts tumor suppressor ${ }^{5}$ and oncogenic isoforms of the Ret receptor tyrosine kinase. ${ }^{6}$ Mammalian Csk is the major cellular inhibitor of the Src family kinases through direct phosphorylation. ${ }^{7}$ In Drosophila, broad loss of dCsk activity led to an increase in Src activity. . $^{2,6}$

We have previously reported effects from the epithelial microenvironment in cells deficient for dCsk. ${ }^{2}$ When dCsk activity was reduced or removed within an entire epithelial region, dCsk cells exhibited ectopic growthattributable to increased cell proliferation and decreased death-but retained epithelial integrity. By contrast, discrete dCsk-deficient patches of cellsbounded by wild-type cells-initiated a migratory and invasive program along the basal extracellular matrix. Such cellular behavior included (1) dissolution of the adherens junctions as assessed by delocalization of junction-associated proteins such as p120ctn, and (2) activation of the Rho GTPase and Jun kinase (JNK) pathway. The metalloproteinase dMmp2 was also required for this $d C s k-d e p e n d e n t$ invasive migration phenotype. $^{2}$

In this study, we present evidence indicating that the remaining member of the Drosophila matrix metalloproteinase (MMP) family, dMmp1, is also required for the invasive phenotype of boundary dCsk cells. Our evidence includes genetic modifier interactions, promoter activation, increased protein levels, and in situ enzymatic activity. Remarkably, sections of human oral squamous cell carcinomas (OSCCs) exhibited a border difference strikingly similar to effects observed in our Drosophila model: human MMP2 was up-regulated at the borders of tumor islands whereas expression of the junctional markers E-cadherin and P120-catenin was decreased or delocalized in these same boundary cells. This indicates that the two models may share some aspects of tumor progression including the importance of local interactions within the epithelium.

\section{Materials and Methods}

\section{Drosophila Genetics}

We previously used the UAS-dCsk-IR, UAS-p120ctn-GFP $U A-R F P[n / s]$, and ptc-gal/4 transgenes as a model for tumorigenesis including metastasis. ${ }^{2}$ The transgenes actin-gal4 and tub-gal80ts and the mutant lines dmmp $1^{\text {kO4809} / C y O ~}$ and $\mathrm{dmmp} 2^{\mathrm{EY} O 8942} / \mathrm{CyO}$ were obtained from the Bloomington Drosophila Stock Center. Note that dmmp2 $2^{\text {EYo8942 }}$ is a UAS-bearing $P$ element insertion and could potentially result in gain of function phenotypes in the presence of gal4; however, it is inserted within the gene region and results in a loss of function allele. dmmp1-lacZ and UASdmmp1-IR transgenes inserted on the second and third chromosome were a gift from M. Uhlirova and D. Bohmann, and UAS-dmmp1.F1 from A. Page-MacCaw. All cultures and incubations were performed at $25^{\circ} \mathrm{C}$ on standard molasses media.

Full genotypes in figures: Figure 1, A-C: ptc-gal4, UASp120ctnGFP, UAS-RFP[n/s]. Figure 1, D-F: ptc-gal4, UASp120ctnGFP, UAS-RFP[n/s], UAS-dCsk-IR.

Figure 2A and sixth box in 2G: ptc-gal4, UAS-GFP[n/s]. Figure 2B and first box in 2G: UAS-dCsk-IR; ptc-gal4, UAS-GFP $[n / s]$. Figure $2 \mathrm{C}$ and second box in 2G: UASdCsk-IR; ptc-gal4, UAS-GFP[n/s]; puc ${ }^{E 69 /+}$. Figure 2D and third box in 2G: UAS-dCsk-IR; ptc-gal4, UASGFP $[n / s] / U A S-d m m p 1-I R .^{2}$ Figure 2E and fourth box in 2G: UAS-dCsk-IR; ptc-gal4, UAS-GFP[n/s]/UAS-dmmp1$I R{ }^{3}$ Figure 2F and fifth box in 2G: UAS-dCsk-IR; ptc-gal4, UAS-GFP[n/s]/dmmp $1^{\text {k04809. }}$

Figure 3A: actin-gal4/+;tub-gal80 ts; Figure 3B: actingal4/+;tub-gal80ts /UAS-dmmp1.F1 Figure 3, C, D, G, and $\mathrm{H}:$ ptc-gal4, UAS-RFP [n/s]; Figure 3, E and F: UAS-dCsk- 

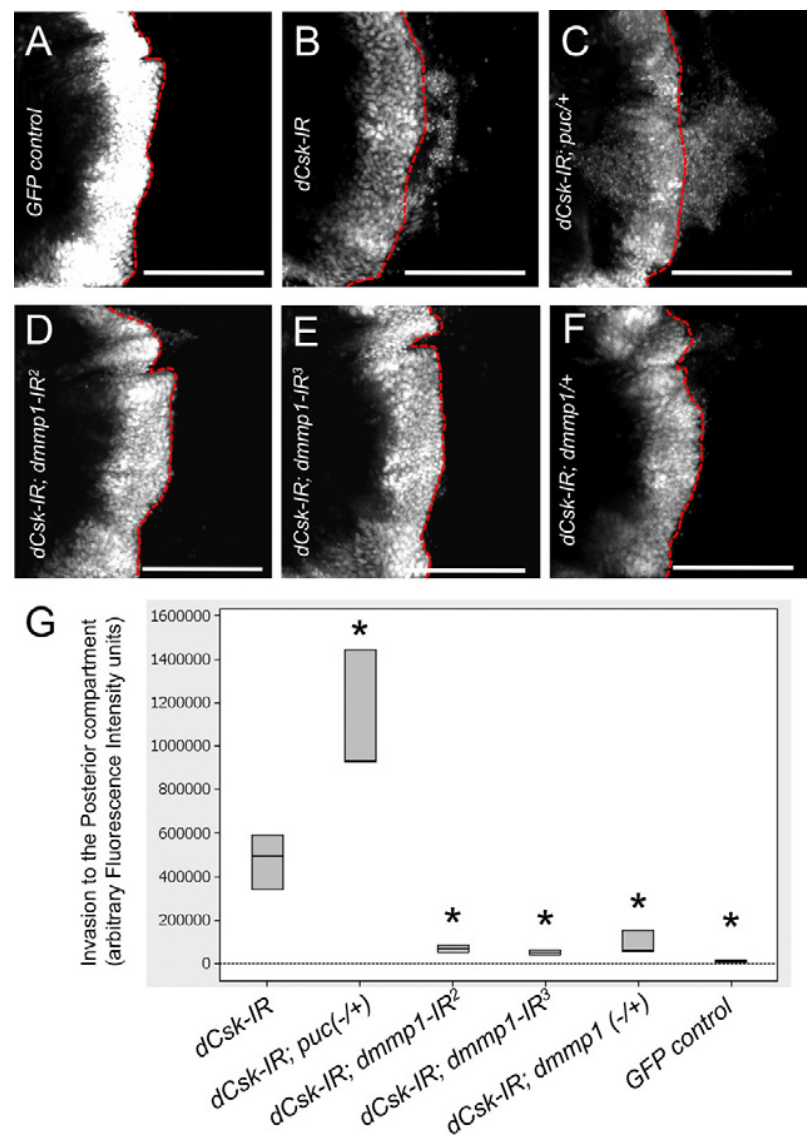

Figure 2. dMmp1 is required for invasion of dCsk-IR cells. Confocal projections of larval wing disks. The ptc region was labeled with GFP (gray). Red dotted lines mark the anterior/posterior boundary. Posterior is toward the right. Scale bars $=100 \mu \mathrm{mol} / \mathrm{L}$. A: Control disks ( $p t c>G F P(n l s)$ showed no cells migrating toward the posterior compartment. B: Reducing dCsk activity ( $p t c>d C s k-I R, G F P[n l s]$ ) led to cell migration. Note the presence of ectopic GFP in the posterior compartment. C: Removal of one functional genomic copy of puckered ( $p t c>d C s k-I R, U A S-G F P[n l s] / p u c^{E 69 /+}$ ) enhanced the migrating phenotype of $d C s k-I R$ cells. $\mathbf{D}$ and $\mathbf{E}$ : simultaneous targeting of dCsk and dMmp1 by RNAi resulted in reduced cell migration. F: Removal of one functional genomic copy of $d m m p 1$ ( $p t c>d C s k-I R, G F P[n l s] / d m m p 1^{k 04809 /+}$ ) also reduced the number of migrating cells. G: Quantification of the invasive phenotype. See Materials and Methods for details. There are statistically significant differences with the reference genotype ( $p t c>d C s k-I R$, first box in the plot; $\left.{ }^{*} P<0.05\right)$ from nonparametric Mann-Whitney tests.

IR; ptc-gal4, UAS-RFP [n/s]; Figure 3, I and J: ptc-ga/4, UAS-GFP[n/s]/dmmp1-lacZ; Figure 3, K and L: UASdCsk-IR; ptc-ga/4, UAS-GFP[n/s]/dmmp1-lacZ.

\section{Histology}

Immunofluorescence was performed as previously described. ${ }^{8}$ The antibodies used were anti- $\beta$-galactosidase (1:100, from Cell Signaling, Boston, MA) and anti-dMmp1 (1:10, from the Developmental Studies Hybridoma Bank). Primary antibodies were visualized with Alexa 532- or 568-conjugated secondary antibodies (Molecular Probes, Invitrogen, Carlsbad, CA).

The in situ zymography technique for Collagenase/ Gelatinase activity was performed as follows: dissected unfixed fly wing imaginal disks were incubated immediately in $100 \mu \mathrm{g} / \mathrm{ml} \mathrm{DQ}$ gelatin (Fluorescein conjugated, Molecular Probes) in PBS for 40 minutes at room temper- ature. Tissues were transferred to fixative solution $(4 \%$ formaldehyde in PBS) for 30 minutes at $0^{\circ} \mathrm{C}$. No background signal was observed in tissues that were fixed before incubation with $\mathrm{DQ}$ gelatin (data not shown).

\section{Quantification of the Invasive Phenotype}

We quantified the invasion phenotype using the following procedure: Apical confocal sections from wing disks from the different genotypes - all containing the ptc-gal, UASGFP transgenes-were used to identify the Anterior/Posterior compartmental boundary. Next, the integrated fluorescence density from the posterior compartment was obtained from full confocal projections by using Image $\mathrm{J}$ (National Institutes of Health) software.

Mann-Whitney statistical tests were performed with all other genetic backgrounds versus the ptc $>d C s k-I R$ reference genotype ( $n=3$ for each genotype).

\section{Human Tumors}

Human tumor studies included 15 patients with OSCC who had given informed consent. Tissue specimens were obtained after surgical resection for the primary tumors. $5-\mu \mathrm{m}$ paraffin sections of human OSCCs were mounted on glass slides and stained with hematoxilin and eosin for histopathological analysis. The corresponding serial sections were probed with immunohistochemistry using the Histostain kit (Zymed, San Francisco, CA) following the manufacturer's instructions. Primary antibodies included anti-MMP2 (Abcam, Cambridge, MA), anti-E-cadherin, and anti-P120-catenin (both from BD Transduction Laboratories, San Jose, CA) diluted 1:100. Samples were examined with a Leica DM1000 microscope and images recorded with a Leica DFC280 camera. Areas with pronounced inflammation or necrosis were avoided. For quantification, the cases were analyzed independently by two trained pathologists (L.S. and L.Y.) and counted if there was at least one positive tumor island per case.

The available clinicopathologic information on each case including age, gender, tumor site, stage, and histological grade was as follows: variable number, 15\%; age range, 42 to $78 \mathrm{yrs}$; gender, male (10) and female (5). Tumor sites included: larynx (6), tongue (4), oral cavity (3), tonsil (2). Stage: I (0), II (3), III (5), and IV (7). Histological grade: poor (2), moderate (9), good (4).

\section{Results}

\section{Migration of ptc $>$ dCsk-IR Cells Required MMP Activity}

We previously observed that the phenotype of genotypically dCsk cells depended on their cellular microenvironment. For example, whole eye clones of dCsk overproliferated and blocked cell death to yield an enlarged eye, whereas smaller, discrete dCsk clones were eliminated by apoptosis. ${ }^{2}$ Similarly, using RNA-interference to reduce $d$ Csk activity ( $d$ Csk-IR) in a discrete region of the 

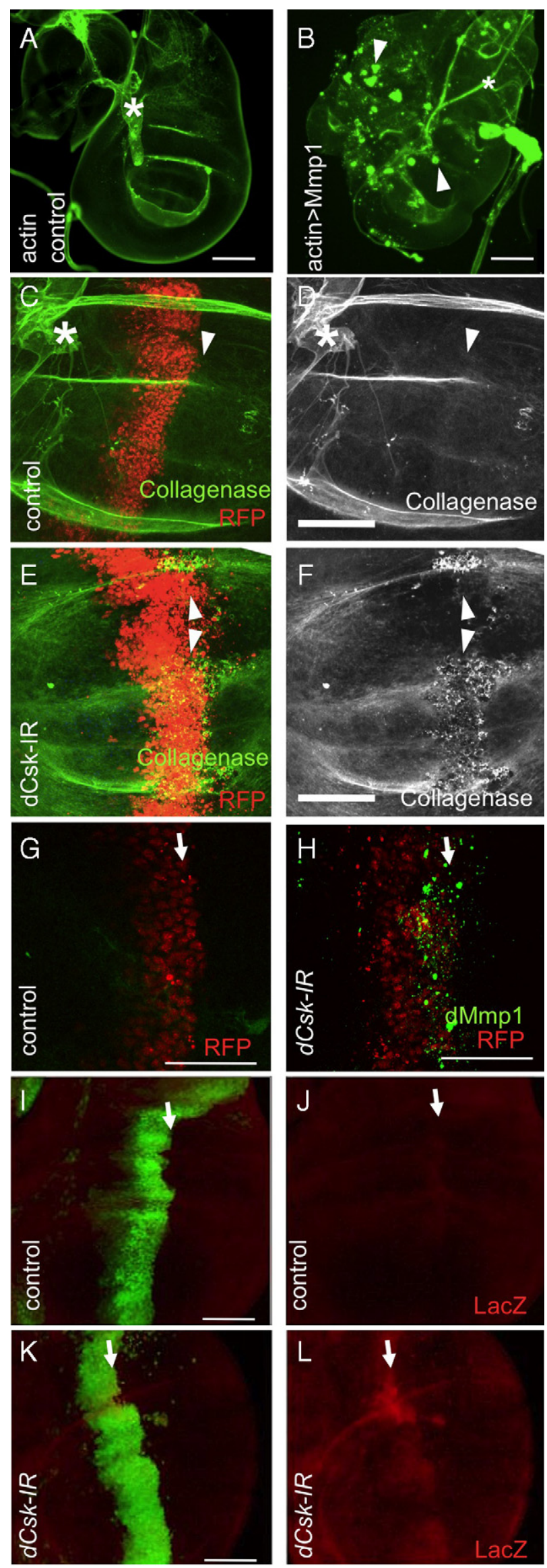

wing led to delocalized expression of the Drosophila P120-catenin ortholog dP120-catenin within the zonula adherens (Figure 1, A-F). ${ }^{2}$ This delocalization of a junctional protein occurred exclusively within $d C s k-I R$ cells at the border between the $d C s k$-IR domain and the neighboring wild-type cells, providing evidence for local interactions between transformed 'boundary' cells and their wild-type neighbors within the epithelium.

In addition to altered zonula adherens, these transformed 'boundary' cells exhibited aspects of invasion. Expressing $d C$ sk-IR in a stripe at the anterior/posterior boundary of the developing wing (ptc $>d C s k-I R$ ), these 'boundary' cells became basally extruded from the epithelium. Once released they migrated along the basal extracellular matrix; eventually, these migrating ptc $>d$ Csk-IR cells expressed activated caspase, most died by apoptosis as assessed by the emergence of pycnotic nuclei (ref. 2 and data not shown). As border cells left the epithelium the newly revealed tumor border renewed the process, resulting in a progression of extruded cells.

This metastasis-like process required a cascade of proteins including the signaling factors dE-cadherin, dP120-catenin, Rho1, and Jnk as assessed by genetic modifier tests. ${ }^{2}$ Migration of ptc $>d C s k-I R$ boundary cells also required the MMP ortholog dMmp2: removal of one functional genomic copy led to suppression of the migration phenotype (ptc $>d C s k-I R ; d m m p 2^{\text {ey08942/- }}$, data not depicted). ${ }^{2}$ Metastatic cell behavior commonly requires the activity of multiple MMP proteins. ${ }^{9}$ The Drosophila genome encodes a second predicted MMP, dMmp1. dMmp1 is commonly under the control of Jnk, ${ }^{10}$ a central mediator of ptc $>d C$ Ck-IR migration. Consistent with this, the invasion phenotype was enhanced in a puckered heterozygous $\left(p_{1} \mathrm{c}^{\mathrm{ES} /+}\right.$ ) background (Figure 2, A-C). The puc locus encodes a negative regulator of dJnk activity, and its reduction permits higher levels of JNK activation. $^{2}$ Nevertheless, it could not be ruled out that the genetic interaction observed with puc ${ }^{E 69}$ was attributable to a background mutation/s.

Figure 3. Increased dMmp1 in dCsk-boundary cells. All images were confocal projections across the tissues, except $\mathbf{C}-\mathbf{F}$, which were confocal sections at epithelial planes. A: Control wing disks (actin-gal; tub-gal80 $0^{t_{5}}$ ) were stained with DQ gelatin to perform in situ zymography for collagenase/gelatinase activity. Asterisks in $\mathbf{A}$ and $\mathbf{B}$ point to trachea branches, a tissue expressing high levels of MMPs. Note the fibrous nature of the collagenase/gelatinase activity staining, which localized to the extracellular matrix basal from the epithelium. B: Ubiquitous expression of dMMP1-by incubating actin>dmmp1; tub gals $0^{t s}$ third instar larvae for six hours at the nonpermissive temperature $\left(29^{\circ} \mathrm{C}\right)$ - resulted in increased collagenase/gelatinase activity in foci across the tissue (arrowheads). C: Control wing disks $(p t c>R F P[n l s])$ stained with DQ gelatin (green). $\mathbf{D}$ and $\mathbf{F}$ show the DQ gelatin staining in grayscale from $\mathbf{C}$ and $\mathbf{E}$, respectively. E: Reducing $d C s k$ activity ( $p t c>d C s k-I R, R F P[n l s])$ led to an increase in collagenase/gelatinase activity biased toward the $\mathrm{A} / \mathrm{P}$ boundary (arrowheads). G: Control disks ( $p t c>R F P[n l s])$ contain low levels of $\mathrm{dMmp} 1$ protein as assessed with an anti-dMmp1 antibody (green), the ptc domain was visualized with RFP[nls] (red). H: Reducing $d C s k$ activity $(p t c>d C s k-I R, R F P[n l s])$ led to a strong increase in expression of $\mathrm{dMmp} 1$ (green) protein biased to the A/P boundary (arrow). I-J: Promoter activity of dmmp1 (dmmp1-lacZ) was visualized in developing wing disks by an antibody to $\beta$-galactosidase (red); the ptc domain was visualized with GFP[nls] (green). Control disks ( $p t c>G F P[n l s], d m m p 1-l a c Z$ ) exhibited widespread low-level $d m m p 1$ promoter expression. K-L: When $d C s k$ activity was reduced ( $p t c>d C s k-I R, G F P[n l s], d m m p 1-l a c Z$ ), levels of $d m m p 1$ expression strongly increased specifically within the boundary of the ptc domain (arrow). Scale bars $=100 \mu \mathrm{m}(\mathbf{A}$ and $\mathbf{B})$ and $50 \mu \mathrm{m}(\mathbf{C}-\mathbf{L})$. 
Importantly and consistent with a role of dMmp1 in migration, removal of one functional genomic copy of dmmp1 (ptc >dCsk-IR; dmmp $1^{k 04809 /+}$ ) strongly reduced $d C s k-I R$-mediated boundary cell migration (Figure 2F). We quantified the invasion phenotype by measuring the levels of ectopic fluorescence in the posterior compartment (Figure 2G, see Materials and Methods). Furthermore, RNA interference transgenes targeting dmmp1 mRNA also suppressed the invasive phenotype of ptc $>d C s k-I R$ cells (Figure 2, D, E, and G). Together, these results indicate that Drosophila dMmp1 is required for the metastasis-like behavior of $d C s k-I R$ boundary cells, including the invasive migration into the posterior compartment.

To further explore the in situ activity of Drosophila MMPs in the presence of ptc $>d C s k-I R$, we developed a zymography technique to assess gelatinase/collagenase activity in cultured Drosophila tissue. Developing wing disks were coated in the enzyme activity reporter DQ-gelatin (See Materials and Methods), which measures cleavage activity by release of fluorescence quenching. MMPs have been implicated in the remodeling of the tracheal branches during larval growth. ${ }^{11,12}$ Consistent with this and serving as an internal control, we observed high levels of gelatinase/collagenase activity in the tracheal branches associated with wing disks (asterisks in Figure 3, A-C). Expressing ectopic dMmp1 in third instar larvae resulted in increased collagenase/gelatinase activity (Figure 3B). This is consistent with the view that dMmp1 possesses collagenase/gelatinase activity as expected, although activation through indirect means cannot be ruled out. Even in the absence of exogenous transgenes, the basal membrane from control disks displayed significant levels of gelatinase/collagenase activity (eg, Figure $3, A, C$, and D). This result suggests that normal developing imaginal disks from Drosophila larvae actively remodel their extracellular matrix, perhaps to accommodate changes in size and shape.

Importantly, endogenous gelatinase/collagenase activity was strongly enhanced in ptc $>d C s k-I R$ disks at the site of expression (Figure 3, E and F). In particular, stained punctae were observed within the basal extracellular matrix that likely represent the 'footprints' of individual invading cells (Figure 3F, e.g.; arrowheads). Together, these results indicate a requirement for activity of both dMmps to mediate ptc $>d$ Csk-IR-dependent migration.

\section{dMmp1 is Controlled at the Level of Transcription}

Dependence on MMP activity was further reflected by an increase in dMmp1 protein expression: levels of dMmp1 protein were greatest at ptc $>d C s k-I R$ boundary cells (Figure 3, G and H). MMP activity can be controlled at multiple levels including transcriptional, posttranscriptional, and posttranslational. ${ }^{13}$ To assess whether dMmp1 is regulated at the level of transcription, we used a reporter transgene that included $d m m p 1$ gene enhancers fused to a minimal promoter (dmmp1-lacZ). ${ }^{10}$ Reporter expression was strongly increased in genotypically ptc $>d C s k-I R$; ptc $>$ GFP; dmmp1-lac $Z$ wing disks versus controls ( $p t c>$ GFP; dmmp1-lac $Z$ ) as assessed with anti- $\beta$-galac- tosidase antibodies (Figure 3, I-L). Taken all together, these results are consistent with the requirement of both Drosophila MMPs in the phenotype of transformed cells at the ptc $>d$ Csk-IR boundary and that regulation of $d M m p 1$ was at least in part through transcriptional control.

\section{Evidence for a Mechanistic Link between Drosophila Tumor Boundary Signaling and Invasion in Human Squamous Cell Carcinoma}

Our studies in Drosophila suggest a model in which transformed cells receive local signals from their normal untransformed neighbors and that these signals lead to (1) junctional changes, (2) activation of MMPs, and (3) metastasis-like invasive migration. This view emphasizes the importance of local cell-cell interactions within the epithelium that mediate release of transformed cells at the tumor boundary. The mechanisms that direct metastasis remain poorly understood, and we next asked whether similar boundary alterations were present in human carcinomas.
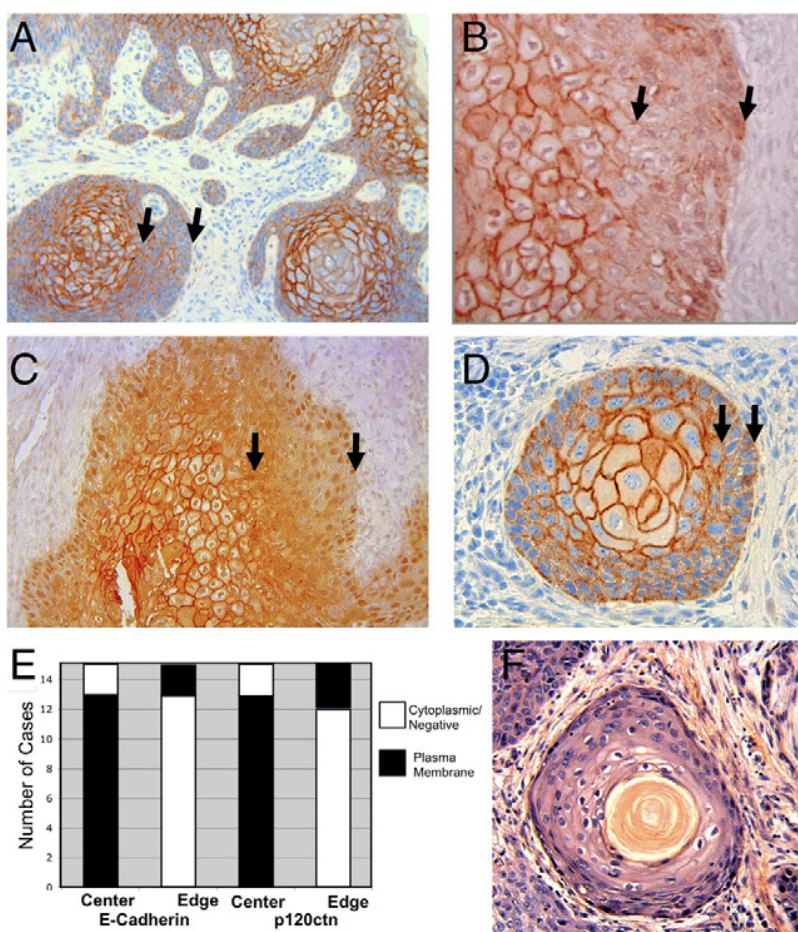

Figure 4. E-cadherin and P120-catenin are reduced or delocalized specifically at the tumor boundaries of human squamous cell carcinomas. Immunohistochemistry of SCCs of the oral cavity (larynx). In Figures 4 through 6, antibody staining is visualized with diaminobenzidine staining (brown-red) in tumor tissue that is counterstained with hematoxylin to visualize nuclei (blue). Original magnifications: $\mathbf{A}$ and $\mathbf{C}, \times 10 ; \mathbf{B}, \mathbf{D}$, and $\mathbf{F}, \times 20$. $\mathbf{A}$ and $\mathbf{B}$ : E-cadherin protein was lost from junctions specifically at tumor borders (area between arrows) as assessed with an anti-E-cadherin antibody. $\mathbf{C}$ and $\mathbf{D}$ : P120-catenin was similarly lost at tumor borders. E: Phenotype frequencies from both antibodies in 15 analyzed specimens. In most specimens, both proteins were strongly localized to the cell-cell junctions (plasma membrane) at the center of the tumor islands but were reduced or delocalized in cells at the tumor borders. F: A hematoxylin/eosin staining from a tumor island containing a 'keratin pearl' in its center. This suggests that some tumor islands could possess an outside-in polarity related to the apical-basal polarity of stratified epithelia. 
The effects of the microenvironment at tumor margins are best observed early in the progression of primary tumors, which retain a coherent boundary. We therefore focused on SCCs of the oral cavity, which are readily obtained as early primary tumors. We used immunohistochemistry to explore human orthologs of gene products implicated in our Drosophila studies. We first examined junctional proteins; Drosophila orthologs of E-cadherin and P120-catenin were lost specifically at 'tumor' boundaries. Although both E-cadherin and P120-catenin were observed at cell-cell junctions throughout most of the human OSCC tumors, both were lost specifically at tumor boundaries in nearly all tumors examined (Figure 4, A-F; see also ref. 14) in a manner strikingly similar to the loss observed in our Drosophila ptc $>d C s k-I R$ model.

In our Drosophila model, migration of ptc $>d C s k$-IR tumors was specific to cells at the tumor boundary as assessed by histology and by border-specific expression of dMmp1. The mammalian genome encodes at least 24 MMPs: Drosophila dMmp2 is structurally related to membrane-anchored mammalian MMPs, whereas dMmp1 is similar to secreted MMPs. Previous mammalian studies indicated that Src activation results in a JNK-dependent up-regulation of MMP2 in cell culture, ${ }^{15}$ and we therefore focused on the secreted protein MMP2; of note, MMP2 is not the closest homolog to $\mathrm{dMmp2}$.

Significantly, we found that MMP2 was consistently expressed at higher levels by cells at the border of OSCC tumor islands compared with the remainder of the tumor (Figure 5, A-F). When consecutive sections from the
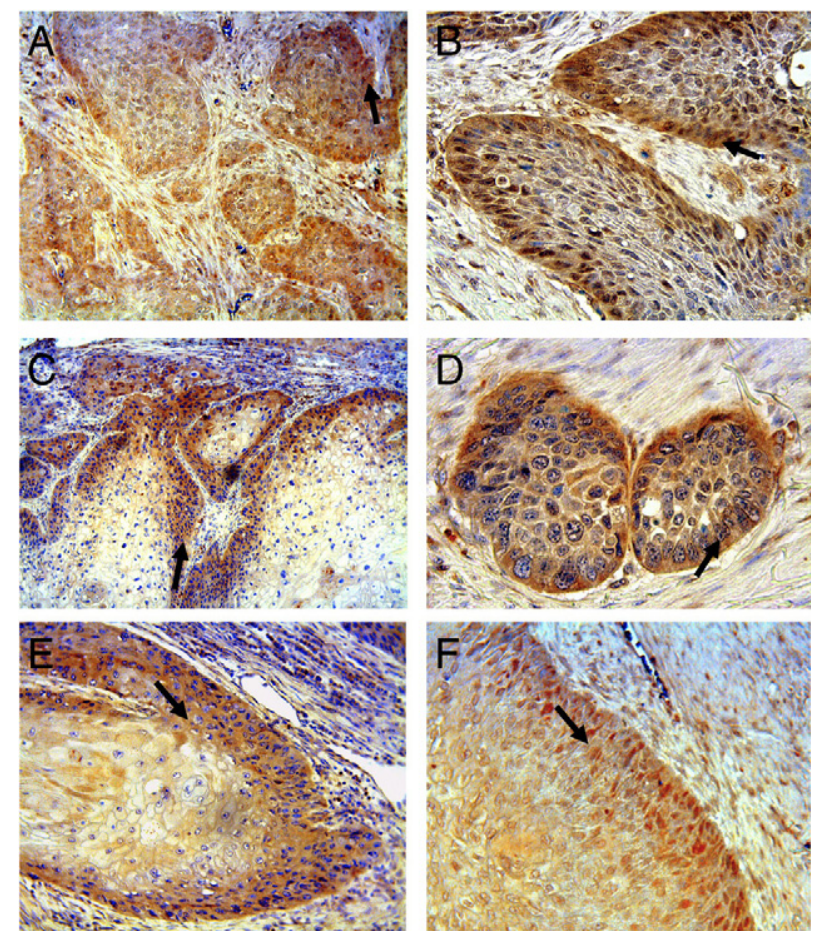

Figure 5. Increased MMP2 expression was observed at SCC tumor boundaries. Sections from patients' OSCCs were stained with antibodies specific to MMP2. Cells at the edges of the tumor islands consistently displayed higher levels of MMP2 protein (eg, arrows). Type and original magnifications: A: larynx $(\times 10)$; B: larynx $(\times 20)$; C: tongue $(\times 10)$; D: larynx $(\times 40)$; E: tonsil $(\times 10)$; and $\mathbf{F}$ : larynx $(\times 20)$.

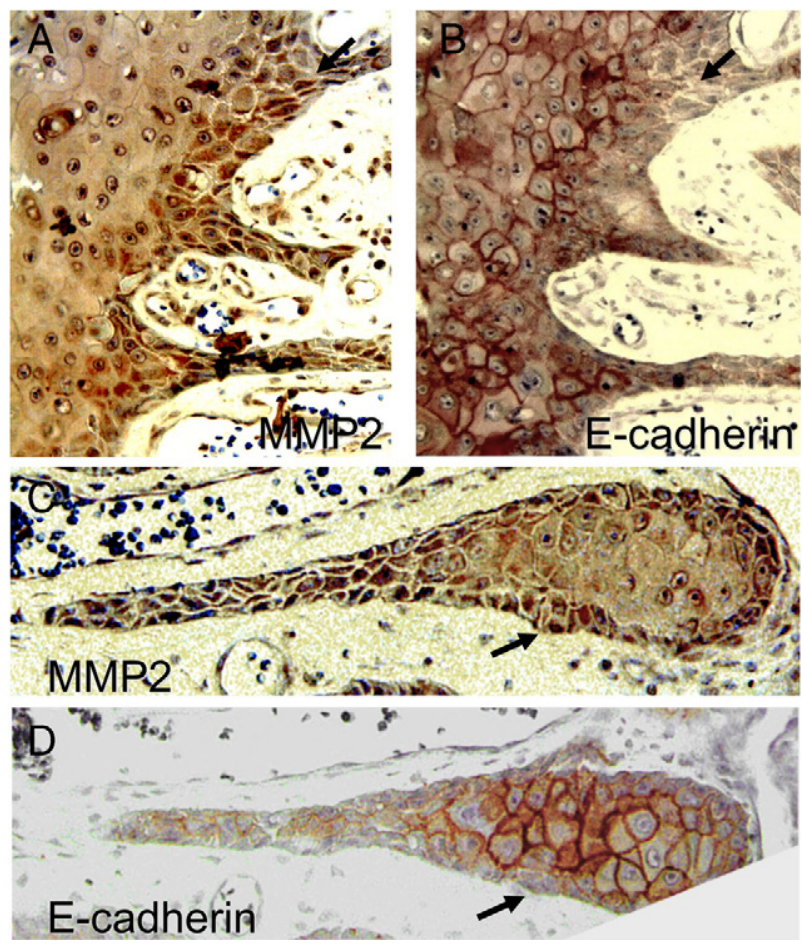

Figure 6. Increased MMP2 expression correlated with reduced or delocalized E-cadherin expression. Abutting sections ( $\mathbf{A}$ and $\mathbf{B})$ and $(\mathbf{C}$ and $\mathbf{D})$ of an oral cavity SCC were stained with antibodies specific to MMP2 and Ecadherin as labeled. The same population of cells at the tumors' boundaries (arrows) consistently demonstrated both increased MMP2 expression and decreased or delocalized E-cadherin. Original magnifications, $\times 20$.

same tumor sample were stained for E-cadherin and MMP2, the results indicated that the same border cell population that displayed reduced or delocalized expression of E-cadherin also expressed increased levels of MMP2 (Figure 6, A-D). These data indicate that mechanisms active in our ptc $>d C s k-I R$ model-local cell-cell interactions that alter junctions and provoke metastasismay account for key aspects of cell shedding and invasion in SCCs.

\section{Discussion}

Metastasis has traditionally been viewed as a relatively late outcome of genomic changes within an evolving tumor. Recently, however, evidence has emerged that cells migrate from early-even benign-tumors. For example, breast tumor cells have been observed within the bone marrow of patients with early stage tumors including Stage 1 and DCIS. ${ }^{16}$ This and similar data ${ }^{17}$ suggest that metastasis is an early event. In this view, both growth and shedding of primary cells occur early in the tumor process. Our own work in Drosophila led to a model consistent with this view, in which local interactions between a tumor's boundary cells and their wild-type neighbors provoke metastasis of the former; we proposed that this process occurs at the earliest stages of tumor emergence and that it does not require additional genomic insults. $^{2}$ In this study, we provide evidence potentially 
linking these views and emphasizing the unique properties of cells at tumor borders.

Understanding the mechanisms by which cancer cells become invasive and eventually reach the circulatory system remains of key importance. MMPs play a key role during this process by providing cells a means to manipulate the extracellular matrix. MMPs have yet to prove a useful target for therapeutics attributable to the abundance of MMP paralogs and the pleiotropic roles they play during development and homeostasis. ${ }^{18}$ Nevertheless, the functional role of MMPs in our ptc $>d$ Csk-IR metastasis model and the correlation between loss of junctional proteins and high MMP expression in OSCCs further emphasize the potential utility of the upstream pathways regulating these proteins as therapeutic targets.

Our Drosophila ptc $>d C s k-I R$ model suggests that the interaction of tumor cells with their wild-type epithelial neighbors is a central regulator of invasion. In human OSCCs, cells typically invade the stromal space as large 'tumor islands.' We found that cells at the borders of these islands commonly displayed reduced or delocalized E-cadherin and P120-catenin expression and increased MMP expression. Based on the similarities with our observations in Drosophila, we postulate that this differential phenotype of cells at the border versus the center of tumor islands represents an 'echo' from their previous interactions with normal epithelial cells while in their site of origin. We further postulate that cells shed from these tumors retain these characteristics as they migrate to distant sites (e.g., see ref. 14).

Additional tumor types will therefore need to be examined to determine whether these border effects are a general phenomenon. Unfortunately, tumor types such as lung and breast are typically diagnosed late in their progression and coherent primary tumors are difficult to obtain. Although SCC tumor islands retain many epithelial characteristics observed in these tumor types, they show some differences from classical epithelial-to-mesenchymal transition modes of invasion in which cells aberrantly activate embryonic developmental programs such as Snail and Twist. ${ }^{19}$

Our data do not exclude other possible models. For example, the different phenotypes from cells at the border versus the center of the tumor island could represent differentiation gradients present from the basal to apical strata within stratified epithelia. The presence of keratin pearls at the center of some tumor islands supports this possibility (Figure 4F). However, we deem this unlikely given the sharp boundary in which cells exhibit junctional and MMP differences. A more likely alternative model is the possibility that these border differences reflect interaction not with epithelial neighbors but with the surrounding stromal environment. This stromal environment provides extracellular matrix components, fibroblasts, macrophages, and other immune cells that are capable of providing localized signals to the tumor. Although the importance of stromal signals has been well established, ${ }^{1}$ this model does not explain how the tumor island initially exited the epithelium and invaded across the basal lamina. Stromal factors are unlikely to contribute significantly to the ptc $>d$ Csk-IR invasion because we observed boundary effects while the cells were still well integrated within the epithelium. Furthermore, Hogan et al recently reported that nontransformed epithelial cells play an important role in the behavior of transformed mammalian cells: in concordance with our fly work, transformed cells delaminated from cultured epithelial sheets only when juxtaposed to nontransformed cells. ${ }^{20}$

Border cells that lost junction-associated E-cadherin and P120-catenin also displayed increased MMP2 expression, suggesting a link between the two. In our ptc $>d C s k-I R$ model, JNK likely provides a link between $\mathrm{dE}$-cadherin/dP120-catenin and MMPs. ${ }^{2}$ Soubry et al observed that the delocalization/reduction of E-cadherin and P120-catenin at tumor borders correlated with subcellular localization of the transcription factor Kaiso. ${ }^{14}$ Kaiso interacts physically with P120-catenin and therefore represents an additional candidate link between P120-catenin and MMPs. Consistent with this view, a recent report demonstrated that $\mathrm{P} 120$-catenin together with Kaiso regulate expression of MMP7 in mammalian gastric cells. ${ }^{21}$ Drosophila does not contain a clear Kaiso ortholog by sequence similarity and a true molecular link in the fly model may still await identification.

In this and our previous study, ${ }^{2}$ we show evidence indicating that both Drosophila MMP orthologs act to mediate the invasive migration phenotype of boundary $d C s k$ cells. We observed an analogous distribution of MMP2 in SCC tumor islands; additional studies will be required to determine whether other MMPs exhibit similar boundary localization. Further studies will also be required to determine whether metastatic cells are shed primarily from mammalian tumor boundaries and to understand the relative contributions of local epithelial cells versus local stroma in this process.

\section{Acknowledgments}

We thank Andrea Page-MacCaw, Mirka Uhlirova, and Dirk Bohmann for kindly sharing fly stocks and the David Ornitz laboratory for initial assistance with immunohistochemistry.

\section{References}

1. Kenny PA, Lee GY, Bissell MJ: Targeting the tumor microenvironment. Front Biosci 2007, 12:3468-3474

2. Vidal M, Larson DE, Cagan RL: Csk-deficient boundary cells are eliminated from normal Drosophila epithelia by exclusion, migration, and apoptosis. Dev Cell 2006, 10:33-44

3. Vidal M, Cagan RL: Drosophila models for cancer research. Curr Opin Genet Dev 2006, 16:10-16

4. Brumby AM, Richardson HE: Using Drosophila melanogaster to map human cancer pathways. Nat Rev Cancer 2005, 5:626-639

5. Stewart RA, Li DM, Huang H, Xu T: A genetic screen for modifiers of the lats tumor suppressor gene identifies C-terminal Src kinase as a regulator of cell proliferation in Drosophila. Oncogene 2003, 22:6436-6444

6. Read RD, Bach EA, Cagan RL: Drosophila C-terminal Src kinase negatively regulates organ growth and cell proliferation through inhibition of the Src. Jun N-terminal kinase, and STAT pathways. Mol Cell Biol 2004, 24:6676-6689

7. Imamoto A, Soriano P: Disruption of the csk gene, encoding a neg- 
ative regulator of Src family tyrosine kinases, leads to neural tube defects and embryonic lethality in mice. Cell 1993, 73:1117-1124

8. Brachmann CB, Jassim OW, Wachsmuth BD, Cagan RL: The Drosophila Bcl-2 family member dBorg-1 functions in the apoptotic response to UV-irradiation. Curr Biol 2000, 10:547-550

9. Rosenthal EL, Matrisian LM: Matrix metalloproteases in head and neck cancer. Head Neck 2006, 28:639-648

10. Uhlirova M, Bohmann D: JNK- and Fos-regulated Mmp1 expression cooperates with Ras to induce invasive tumors in Drosophila. EMBO J 2006, 25:5294-5304

11. Page-McCaw A, Serano J, Sante JM, Rubin GM: Drosophila matrix metalloproteinases are required for tissue remodeling, but not embryonic development. Dev Cell 2003, 4:95-106

12. Page-McCaw A: Remodeling the model organism: matrix metalloproteinase functions in invertebrates. Semin Cell Dev Biol 2008, 19:14-23

13. Mott JD, Werb Z: Regulation of matrix biology by matrix metalloproteinases. Curr Opin Cell Biol 2004, 16:558-564

14. Soubry A, van Hengel J, Parthoens E, Colpaert C, Van Marck E, Waltregny D, Reynolds AB, van Roy F: Expression and nuclear location of the transcriptional repressor Kaiso is regulated by the tumor microenvironment. Cancer Res 2005, 65:2224-2233
15. Hauck CR, Hsia DA, Puente XS, Cheresh DA, Schlaepfer DD: FRNK blocks v-Src-stimulated invasion and experimental metastases without effects on cell motility or growth. EMBO J 2002, 21:6289-6302

16. Husemann $Y$, Geigl JB, Schubert F, Musiani P, Meyer M, Burghart E, Forni G, Eils R, Fehm T, Riethmuller G, Klein CA: Systemic spread is an early step in breast cancer. Cancer Cell 2008, 13:58-68

17. Podsypanina K, Du YC, Jechlinger M, Beverly LJ, Hambardzumyan $\mathrm{D}$, Varmus $\mathrm{H}$ : Seeding and propagation of untransformed mouse mammary cells in the lung. Science 2008, 321:1841-1844

18. Egeblad M, Werb Z: New functions for the matrix metalloproteinases in cancer progression. Nat Rev Cancer 2002, 2:161-174

19. Yang J, Mani SA, Weinberg RA: Exploring a new twist on tumor metastasis. Cancer Res 2006, 66:4549-4552

20. Hogan C, Dupre-Crochet S, Norman M, Kajita M, Zimmermann C, Pelling AE, Piddini E, Baena-Lopez LA, Vincent JP, Itoh Y, Hosoya H, Pichaud F, Fujita Y: Characterization of the interface between normal and transformed epithelial cells. Nat Cell Biol 2009, 11:460-467

21. Ogden SR, Wroblewski LE, Weydig C, Romero-Gallo J, O'Brien DP, Israel DA, Krishna US, Fingleton B, Reynolds AB, Wessler S, Peek RM Jr: p120 and Kaiso regulate Helicobacter pylori-induced expression of matrix metalloproteinase-7. Mol Biol Cell 2008, 19:4110-4121 